\section{Palobra}

ACCESO $\partial$ ABIERTO

Cómo citar: Jaramillo, B. y Pineda, C. (2020). Significación sobre la diferencia entre violencia y conflicto escolar: un estudio de caso sobre sujetos que han sufrido o generado violencia. Palobra, 20(2), 230-250.

10.32997/2346-2884-vol.20-num.2-2020-3317

Recibido: 23 de julio de 2020 .

Aprobado: 21 de septiembre de 2020 .

Autor de correspondencia:

Bairon Jaramillo Valencia

bairon.jaramillova@amigo.edu.co

Editora: Rosario Blanco Bello. Universidad de Cartagena-Colombia.

Tipología IBN Publindex:

Artículo de Investigación Científica.

\title{
Significación sobre la diferencia entre violencia y conflicto escolar: un estudio de caso sobre sujetos que han sufrido o generado violencia ${ }^{1}$
}

\author{
The meaning in the difference between violence and school \\ conflict: a case study about subjects who have suffered or \\ generated violence
}

Bairon Jaramillo Valencia

Universidad Católica Luis Amigó, Colombia, bairon.jaramillova@amigo.edu.co

Claudia Maria Pineda Taborda

Universidad Católica Luis Amigó, Colombia, cmpineda67@hotmail.com

\section{RESUMEN}

Este artículo tiene como propósito establecer una mirada diferente sobre la importancia de entender quiénes son los sujetos que participan en el acto escolar, en el conflicto y en la violencia escolar; y de igual forma, develar que los fenómenos 'violencia' y 'conflicto escolar' son diferentes a partir de las significaciones que construyen ambas nociones desde una perspectiva hermenéutica. A su vez, la investigación base para la escritura de este texto se ejecutó bajo un paradigma cualitativo con enfoque hermenéutico y tipo de investigación estudio de caso, a través de técnicas de recogida como: encuestas, entrevistas semiestructuradas y grupos focales. Finalmente, este estudio se llevó a cabo en la Escuela San José de los Hermanos Terciarios Capuchinos sede Copacabana-Antioquia, con la participación de la Universidad Católica Luis Amigó-Medellín. Sobre los hallazgos más significativos, se resaltan la no claridad percibida por los participantes hacia lo entendido por violencia y conflicto, al igual que la confusión sobre forma, contenido y características de dichos actos. Finalmente, a manera de conclusión se determina que no se halla una forma clara y definida de la expresión 'violencia', refiriendo el vocablo amplio o restringido como el más interesante.

Palabras clave: conflicto; violencia; escuela; estudiante; sociedad contemporánea.

Copyright: () 2020. Jaramillo, B. y Pineda, C. Este es un artículo de acceso abierto, distribuido bajo los términos de la licencia https://creativecommons.org/licenses/by-nc$\underline{\mathrm{sa} / 4.0 / \text { la cual permite el uso sin restricciones, distribución }}$ y reproducción en cualquier medio, siempre y cuando que el original, el autor y la fuente sean acreditados.

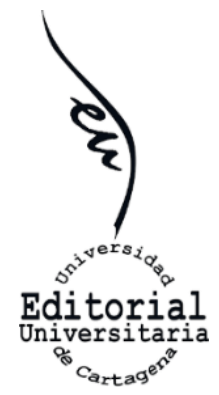

\begin{abstract}
The following article has the purpose of showing a different perspective about the importance of understanding the matter that surrounds the subjects who participate in the school act, in the conflict and in the school violence; likewise, the intention is to unveil that all issues about 'violence' and 'school conflict' are different, based on the meanings that both notions build from a hermeneutic perspective. On the other

\footnotetext{
${ }^{1}$ Este escrito es derivado de una investigación que llevó como nombre "Caracterización de sujetos, prácticas y enfoques de gestión; en la resolución de conflictos en escenarios educativos, I. E. Escuela de Trabajo San José", financiado y ejecutado bajo el apoyo de la Universidad Católica Luis Amigó y la Escuela San José de los Hermanos Terciarios Capuchinos sede Copacabana, desde el año 2016 hasta el 2018.
} 
hand, the inquiry that supported this text was performed under a qualitative paradigm with a hermeneutic approach as well as a case study type of research. The data collection techniques that the research used were: The survey, the semi-structured interview and the focus group; consequently, this study was developed in Escuela San José de los Hermanos Terciarios Capuchinos, Copacabana - Antioquia headquarters, with the collaboration of Universidad Católica Luis Amigó-Medellín. Among the most remarkable finding, it is highlighted the difficulty when the research participants perceived the meaning of violence and conflict, as well as the confusion about form, content and characteristics of the corresponding behaviors. Finally, as a conclusion it is determined that there is not a clear and defined way of explaining the expression 'violence', referring on the other hand the broad or restricted word as the most interesting.

Keywords: conflict; violence; school; student; contemporary society.

\section{INTRODUCCIÓN}

En el mundo de hoy, la violencia escolar parece haberse hecho común, cotidiano, normal; se definió como un asunto de la edad, e incluso como algo connatural al ser humano. En este contexto, se dieron cualquier clase de explicaciones frente a la ocurrencia, frecuencia y gravedad del fenómeno, como la ausencia de compromiso o la falta de un sujeto que se identifique como responsable del hecho; la carencia de indagación y del cuestionamiento crítico de algunos académicos; la incoherencia en el discurso, entre otras. En la actualidad, todo acto de agresión parece estar justificado, pues todo se considera "normal", sin trascendencia y sin importancia. Por ello, no fue extraño que la gran mayoría de los autores consultados no dieran ninguna importancia al hecho de indagar si los fenómenos de violencia y conflicto eran objetos de igual contenido y características diferentes; y, por ende, en la mayoría de los casos, sus conclusiones parecieron ser confusas, inconducentes, inapropiadas o nada contribuyentes a la solución del fenómeno. Así pues, para no incurrir en lo mismo, este texto parte de diferenciar el conflicto del acto violento, sus características, sus sujetos y sus etapas, pero esto no significa negar la importancia del aporte del maestro Olweus (1989), pues, aunque no definió la violencia escolar, destacó una de sus formas con un ejemplo; significó la violencia como un acto o una acción negativa que se genera de forma repetida contra un alumno. El maestro fue claro al calificar la conducta como algo negativo que se hace sobre otro sujeto y contra su voluntad.

En este sentido, es el gran aporte de la observación del maestro Olweus (1989) lo que ha reflejado una primera definición conceptual, bastando un simple análisis de contexto para traducir su ejercicio, este fue claro; existe violencia que se manifiesta con fuerza física y está sucediendo entre escolares, y sucede con una intención clara y preocupante que es causar daño al otro. 
Y es esa intencionalidad de generar daño al otro -la que debe llamar la atención de los académicos, para realizar una investigación rigurosa del fenómeno- lo que suscita la negativa de que el tema de la violencia escolar no puede seguir siendo expuesto con argumentos baladíes, como el que pretende definir la violencia como un hecho connatural al hombre o como una situación necesaria en las relaciones en comunidad.

Es necesario entender que el conflicto y la violencia no son inherentes al hombre o al desarrollo del ser humano, son formas de elección del individuo que están afectando su desarrollo personal, social; y, además, que permean contextos sociales como los centros de formación, aprendizaje o la escuela.

A raíz de la anterior contextualización en lo concerniente al planteamiento del problema, se constituye como propósito dar respuesta a la pregunta: ¿Cuáles son las diferencias que existen entre los vocablos conflicto y violencia, y cómo se definen quiénes son los sujetos que participan en estos actos? Por consiguiente, como objetivo de la investigación, se definió el hecho de posibilitar una visión divergente ante la comprensión de quiénes son, de forma amplia e inclusiva, los actores que participan e intervienen en los actos de violencia y conflicto escolar en el escenario seleccionado para este estudio; asimismo, se plantea dilucidar que el conflicto escolar y la violencia son comportamientos que se relacionan, pero que más allá de dicha conexión, ambos tienen una diferencia marcada en términos hermenéuticos.

Sobre lo que se presentará a lo largo de este artículo, se tiene el esquema de un texto producto de investigación, en el cual se presenta una introducción que fija el propósito, la estructuración y la apertura de los elementos estructurales. Luego se tiene la sección de revisión de la literatura o marco conceptual, donde se definen ampliamente los términos fundantes del estudio, a través de una mixtura entre autores clásicos y contemporáneos.

Acto seguido, se muestra el diseño o estrategia metodológica; representada en el $A, B, C$ del estudio, que sirvió como soporte para la redacción del presente texto. Posteriormente, se da a conocer la sección de resultados y discusión, segmento que desarrolla los hallazgos de forma problematizada, a la luz de la crítica investigativa; y finalmente la sección de conclusión, dedicada al cierre del texto con base a los resultados obtenidos.

\section{Revisión de la literatura}

Para comenzar a desarrollar esta investigación, se debe dejar sentado que las palabras abordadas aquí como vocablos de contenido y características diferentes, se relacionan y complementan; e igualmente, han tenido evolución y han mutado a través del tiempo. 
Figura 1. Categorías conceptuales que asistieron el proceder del estudio.

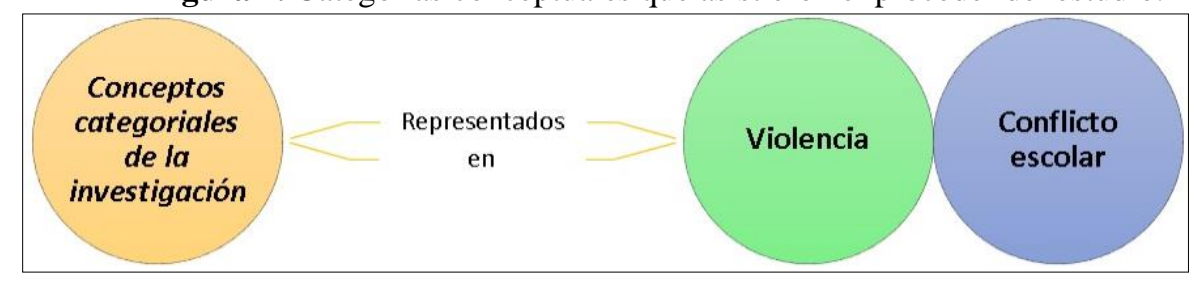

Fuente: Elaboración propia.

\section{Sobre la violencia y la violencia escolar}

Estos dos términos que a veces se utilizan de forma ligera como si fueran sinónimos -y que incluso algunos autores los abordan sin definirlos, sin limitarlos, sin ubicarlos en un contexto claro y preciso- han sido tratados con aparente indiferencia:

La mayoría de las investigaciones sobre violencia escolar se centran, fundamentalmente, en el estudio del fenómeno conocido como bullying, traducido como "acoso escolar entre iguales. Sin embargo, es sólo uno de los tipos de violencia interpersonal que acontecen en los centros educativos, y aunque se le ha puesto mayor atención, también existen otros que por definición no entrarían en el bullying, pero que pueden tener consecuencias igualmente graves, como la violencia interpersonal en los centros educativos o la violencia de género (entregéneros e intergéneros) (Ayala, 2015, p. 494).

Lo antes expuesto nos conduce por coherencia a destacar que la mayoría de las investigaciones parecen iniciar explicando las formas del fenómeno antes que buscar una definición del problema, por ello se hace necesario un estudio más cercano al problema por su gran complejidad; así lo señalan Jano, De Caso y Navas (2012) al afirmar que:

Se puede constatar el grave problema al que nos enfrentamos con la violencia escolar, el cual se especifica día a día en nuestros centros educativos por lo que es necesario tener el máximo conocimiento y recursos para el trabajo en miras a su solución (p. 723).

Es tal la evolución de la sociedad, que hoy figuras como: escuela, familia y sociedad tienen nuevos componentes, por ejemplo hoy se tienen familias compuestas por padres heterosexuales y por padres homosexuales, con y sin hijos, se tienen padres divorciados, sin divorciarse, casados, solteros, en uniones libres; se tienen escuelas de formación virtual y escuelas intramurales, se tiene formación grupal y educación individual; es decir, el individuo ha tenido que evolucionar en la medida que su mundo ha evolucionado, y ha tenido que adaptarse a estos nuevos conceptos independiente de que lo entienda o no. Ese es el mundo actual, una multiplicidad de individuos y de conductas particulares (Sinning, 2006). En este mundo moderno sigue existiendo el concepto de escuela ahora en una 
nueva modernidad, y allí se siguen generando relaciones entre sujetos que unidos por este contexto (el escolar)- deberán convivir en la mejor armonía posible, lo cual no siempre es lo que se vive, de allí la necesidad de entender en este mundo nuevo, cuando comienza la violencia; en este orden de ideas, hay teorías que afirman esta (la violencia escolar) radica en la existencia misma de la escuela, así:

Por otra parte, se encuentra la existencia de la violencia escolar, la cual se produce en la institución educativa, producto de procesos antidemocráticos provocados por el propio sistema educativo. Entre ellos se puede citar: el empleo de metodologías autoritarias, las luchas de poder entre estudiantes, docentes y personal administrativo, el elevado número de estudiantes que impide la personalización de la tarea educativa y la falsa moralidad que ha generado discriminaciones de raza, culto, género (Artavia, 2012, p. 20).

Una muestra evidente de que no existe unanimidad conceptual en el término se puede apreciar en las siguientes afirmaciones: se entiende por violencia escolar como una problemática poliforma y multidireccional, en la cual intervienen todos aquellos que forman parte de la comunidad educativa (maestros, alumnos, directivos, etc.) (Prieto, Carrillo y Jiménez, 2005). Además de este, otro ejemplo se presenta:

La violencia interpares es un grave problema en toda la región; los estudiantes que sufrieron violencia de sus iguales alcanzaron un desempeño en lectura y matemáticas significativamente inferior al de quienes no la experimentaron; en aulas con mayores episodios de violencia física o verbal los educandos muestran peores desempeños que en aulas con menor violencia (Chávez, 2018, p. 2).

Estas citas destacan formas personales de observar el problema desde miradas diferentes; en el uno se quiere evidenciar cómo la violencia escolar puede afectar el rendimiento académico del estudiante; y en el otro, entre quienes sucede. Sin embargo, revisados ambos textos no se puede encontrar una definición de violencia escolar que permita afirmar o entender a qué acto violento se refiere, en ambos textos refieren -como es de esperarse- a la definción del maestro Olweus, y a las diferentes formas en que se evdiencia la violencia. Y aunque es claro que el enfoque de cada investigación es diferente, en lo que se quiere llamar la atención es que ninguna define con claridad el objeto de investigación que es la violencia escolar, pero sí establecen lo que esta causa en clave de escuela, por ello es claro que los enfoques de investigación frente al fenómeno son tan diversos y tan amplios, que pareciera que cada quien puede enfilarse en una teoria de otro o construir la propia (Torres, Palermo y Bedoya, 2018).

Es entonces claro que se está analizando un fenómeno de cuyos orígenes se remontan a unos treinta años atrás; es decir, a inicios de los ochenta, cuando apenas se estaban desarrollando herramientas tecnológicas y los conceptos de 
familia y escuela eran un tanto conservadores, pues solo se concebía en cuanto a la familia conformada por un hombre y una mujer; y en cuanto a escuela, esta estaba en algunos países dictaminada solo para varones; de allí que si regresando al maestro Olweus, se recuerda que sus observaciones suceden entre 1980 y 1998. Es claro que estas distarán bastante alejadas de las observaciones que otro académico pueda realizar en los albores del año 2000 o, incluso hoy, por todos los cambios que se han dado en la historia de la humanidad: cambios políticos, culturales; de conceptos de conformación de familia, de concepto de conformación de escuela, como se precisó en parráfos anteriores. Por ello, la explicacion de Chuqilin y Zagaceta parece aportar algo de claridad por cuanto indica que estos autores vienen manejando diferentes explicaciones del problema, precisando dos posturas en particular: una que llamaron "Restringida" y otra que llamaron "Amplia", obsérvese:

Uno de los principales ejes en la discusión es la confrontación entre los razonamientos que postulan una definición restringida de la violencia y aquellos que plantean una definición amplia de esta misma. En el primer caso, la tendencia es acotar la definición al uso de la fuerza para infligir daño físico grave hacia otros. La perspectiva amplia, en cambio, considera que la violencia física es solo una de las múltiples formas que la violencia puede asumir. Que existen otras formas sutiles, como la violencia simbólica y emocional, u otras manifestaciones que son vividas traumáticamente por las víctimas (Chuquilín y Zagaceta, 2017, p. 2).

Aunque la anterior definición deja ver con claridad las dos escuelas en las cuales pueden circunscribirse los autores según su propia mirada, Álvarez, et al. (2013) hablan del "carácter complejo y multicausal del fenómeno" ( $\mathrm{p}$. 212). Sin embargo, estudiada esta definición con la ya citada de Chuqilin y Zagaceta, es claro que se trata de una definición amplia, que precisa que la violencia escolar tiene múltiples razones y causas, posición que coincide con la propuesta de Flores y Retamal (2011):

La violencia escolar no debe ser rápidamente judicializada o criminalizada, sino comprendida desde claves socioeducativas y desde la misma gestión del conocimiento y los aprendizajes. Preocuparse de la violencia escolar es ante todo insistir en la necesidad de avanzar de un sistema que exige antes que nada rendimiento y performance a uno que exige a los estudiantes articular redes de comprensión del mundo y del sentido de la vida (p. 335).

Es decir, estos autores también se instalan en la definición amplia del fenómeno ya citado, mientras que, para otros autores, el problema conceptual debe ser mirado de manera más integral:

Varios de los estudios muestran que la violencia escolar no puede tratarse como algo generado exclusivamente por individuos problemáticos, sino como un asunto complejo que involucra a diversos 
actores de la comunidad educativa y que debe tratarse con programas integrales que busquen influir en las dinámicas de grupo, en el clima de aula, en el clima escolar y en el clima familiar (Chaux, 2011, p. 84).

Hasta este momento, solo se ha evidenciado la ausencia de unanimidad entre los académicos para definir el concepto de violencia; y en el mismo sentido, se destacan algunas de las miradas particulares del tema desde la perspectiva amplia. Pero desde la visión restringida, las definiciones que contienen alusiones a la fuerza o a la transgresión -como se ha afirmado desde hace más de una década- llegan a ser reducidas ante la necesidad de una comprensión específica:

Se entiende por violencia a la conducta o conductas en las que se hace un uso inadecuado del poder con el objetivo de conseguir dañar a alguien quedando por encima de esa persona, y por supuesto, sin ser consciente de las consecuencias que pueden acarrear las conductas violentas (Aznar, Cacares y Hinojo, 2018, p. 165).

Sin embargo, no basta definirla desde la fuerza, se requieren otros elementos a fin de realizar una definición en contexto real; así pues, Gómez (2013) señala que "la violencia escolar, que no es explicable por sí sola ni se pretende su comprensión viéndola como un fenómeno psicológico individual, sino buscando encontrar su raíz en las formas de organización que presenta la misma institución educativa" (p. 851). Es decir, la violencia escolar tiene sus propias características y sus propios elementos; componentes que inician en la descripción de la fuerza como factor fundante de la definición de violencia escolar, soportada en argumentos múltiples que van desde la no aceptación de la norma escolar, hasta las diferencias personales y de género.

\subsection{Desprendiendo al conflicto de la violencia}

Como se ha indicado en párrafos anteriores, y como bien se ha podido observar, se sigue refiriendo al fenómeno de la violencia sin ser definido. No obstante, hay autores que buscan el origen del problema, entre ellos, Rodríguez, Solá y López (2017), quienes explican que:

El conflicto tiene su origen en un hecho, palabra o situación que ha provocado un malentendido entre dos o más personas. Sin embargo, si lo tomásemos como un combate, lucha o pelea, tales palabras llevan consigo de manera implícita la acción de violencia, al igual que su siguiente definición (p. 103).

Este aporte es muy importante, puesto que inicia una diferencia entre ambas nociones; explica conflicto como "una mala interpretación de algo", y violencia como "un acto de fuerza".

Aunado a ello, otros autores precisan esa diferencia, por ejemplo: 
El conflicto se presenta desde un nivel micro (personas, familia, escuela) hasta el nivel macro (sociedad, estados), y es que este genéricamente, se refiere a cualquier diferendo o desacuerdo, que se manifesté en cualquier momento y/o situación; para su expresión se requiere de dos partes relacionadas (ya sean individuos, grupos, comunidades, o estados - nación) divididas por causas de intereses u objetivos percibidos como incompatibles (Arellano, 2007, p. 31).

Todo ello permite vislumbrar que mientras que la violencia parece tener múltiples enfoques, el conflicto si tiene una definición un poco más clara, más precisa; se conoce como la diferencia o el desacuerdo entre varios sujetos de un mismo contexto social. Al respecto, Cavas (2009) manifiesta que:

Estos conflictos pueden variar ampliamente en cuanto a su intensidad y sus consecuencias dependiendo del modo en que se afronten. Así, en algunos casos estos conflictos son el punto de partida para situaciones graves de violencia escolar, mientras que en otros casos pueden ser una oportunidad para mejorar la relación entre dos alumnos (p. 15).

Es claro que las situaciones que involucran tanto la violencia como el conflicto escolar se presentan en situaciones puntuales, definidas con base al fenómeno que se estudia, entendiendo que existe conflicto como desacuerdo en un contexto escolar y que igual puede darse en un contexto social, pero no como fenómenos naturales al hombre, sino como un factor de convivencia de unos con otros; en un centro común que se llama escuela, en la cual participa un grupo complejo y diverso de individuos, sometidos a unas normas comunes que a veces entienden y aceptan, y otras veces no.

El hecho de que el fenómeno involucre a quienes serán los ciudadanos que conformen las familias, base de cualquier Estado, genera una preocupación no solo en los estamentos escolares sino en toda la comunidad, de donde nacen iniciativas que tienen por intención resolver los conflictos a fin de evitar que ellos concluyan en actos violentos, estas iniciativas deben verse reflejadas en la escuela como un elemento común, pero también en la sociedad que rodea a la escuela; y en términos generales, debe incluir a la institucionalidad (Bonacera, 2015). Por ello en Colombia, se han creado diferentes mecanismos alternativos para la solución de conflictos entre los que es importante destacar la Ley 640, año 2001, institución que pretende dirigir a los ciudadanos a resolver sus diferencias de forma concertada ante un tercero llamado conciliador, quien tiene el deber de escuchar a las partes y ayudarlas a resolver sus diferencias sin tener que acudir ante los jueces de la república.

Estas figuras de carácter estatal, buscan hoy implementarse entre los escolares con la intención de que ellos -mediante diálogos respetuosos y ante terceros que la comunidad escolar identifique como sujetos probos- puedan resolver sus diferencias evitando que se llegue a situaciones de violencia; es decir, 
hoy desde el Estado se busca la intervención del conflicto y la transformación de este hecho negativo en una situación positiva, que puede detenerse a través de una correcta intervención, siendo esta la razón por la cual se encuentra en las escuelas, figuras como: el personero escolar, que tiene como pretensión construir desde la institucionalidad, formas de intervención del conflicto entre sus partícipes sujetos; que al igual que las palabras violencia y conflicto, deberán develarse y explicarse para también intervenirse de la mejor forma posible. Colocados entonces en contexto, es evidente que los fenómenos de violencia y conflicto son situaciones que se presentan entre sujetos que tienen en común el espacio escolar y de allí emana la necesidad de entender quiénes son los sujetos que participan en esos actos, cómo han sido definidos y cuáles son sus características.

Identificadas, conflicto y violencia escolar como fenómenos distintos, necesario se hace saber entre quiénes suceden estas conductas y cuáles son los sujetos que participan en ello, siendo así evidente que los sujetos serán los que se encuentren en contexto escolar; es decir, docentes, estudiantes, administrativos, grupos de apoyo como asociación de padres de familia, psicólogo, entre otros. Por lo tanto, no se puede pensar que los sujetos en contexto escolar son solo profesor y alumno, o viceversa; la escuela es un núcleo en el cual -en razón de un objetivo claro y especifico cual es el aprendizaje- confluyen múltiples sujetos que van desde el portero o el conductor de bus, hasta la señora que administra la tienda de la escuela, pero todos son una comunidad de adultos que están de frente a unos sujetos en formación, y que deben manejar un mismo norte según los programas de cada unidad escolar, por ello en este trabajo se ha de entender como sujeto escolar, a todos los sujetos que conforman el contexto escolar.

Es por lo anterior que se puede aceptar la clasificación general de sujetos de la violencia, en víctimas y agresores, según la cual cada posición puede ser ocupada en forma indistinta por un par, un administrativo, un docente, o un miembro de un grupo auxiliar; tal y como lo señaló desde hace muchos años el maestro Olweus, sin embargo, en los últimos años esta clasificación viene evolucionando. En este sentido, Santoyo y Frías (2014) describen que "la literatura apunta la existencia de dos tipos adicionales: las víctimasagresoras y los observadores" (p. 18). Por consiguiente, ahora los sujetos que se encuentran en el contexto escolar frente a los conflictos o los actos violentos, pasaron de ocupar dos posiciones (víctima - Agresor), a ocupar tres posiciones (víctima - agresor - observador); relaciones en las cuales unos son activos, otros pasivos, así: Agresor (activo) - víctima (pasiva - observador "pasivo/activo").

Es decir, el sujeto agresor será siempre el activo; la víctima que soporta será pasiva, y el observador puede ser activo o pasivo, dependiendo si asume una posición de apoyo a alguno de los sujetos que participan en el acto, como víctima o como agresor, o sí simplemente observa y se complace con lo que 
allí sucede por cualquier razón: identidad con alguna de las partes o indiferencia absoluta frente al hecho.

Algunos autores subclasifican a los sujetos desde su posición; activa o pasiva, advirtiendo que se puede ser víctima y agresor a la vez y por cualquier razón; verbigracia, ser un excelente estudiante, tener una contextura física diferente, un acento diferente, un credo diferente; entendiendo que el agresor es el sujeto que abusa de los demás y la victima quien soporta la agresión, por ello algunos autores han definido al agresor como un sujeto temeroso, al que le hacen falta muchas cosas, cariño, autoestima, valores, apoyo de sus progenitores, etc.

Así pues, los resultados nos permiten concluir que, los agresores presentan un perfil específico frente a las víctimas en general, resultando relevante que todos sean chicos, que destacan por su fortaleza física, su alto grado de provocación y su carácter de cierto liderazgo frente a las víctimas que en su mayoría son chicas, se les considera débiles y cobardes y despiertan un amplio sentimiento de antipatía. Además, entre éstos se aprecia un importante número de alumnos de procedencia extranjera, que puede estar relacionado, en ocasiones, con los índices de rechazo manifestado (Cerezo, 2015, p. 58).

Si se observa con cuidado, se puede establecer que tanto agresor como víctima tienen elementos en común, el más general de ellos parece ser el miedo al rechazo por los otros partícipes del acto escolar, pues el uno actúa para ser reconocido (el agresor), y la víctima sufre la agresión porque no es reconocida o captada, y teme enfrentarse a los demás; sin embargo, "las dificultades escolares se encuentran presentes en agresores y víctimas y, en ambos casos, estas afectan negativamente su rendimiento académico" (Pérez, et al., 2014, p. 76). Lo que de suyo permite afirmar que, en uno y otro caso, su conducta agresiva o de víctima se va a reflejar en los resultados escolares. Ahora, no son solo estos los sujetos que participan en el acto violento o en el conflicto como se deja señalado en párrafos anteriores, también están los terceros, los observadores, los otros, los que no hacen nada, o los que sin querer están tomando partido, sea de forma positiva o negativa, en este sentido se afirma que:

El enfoque del rol participante en el observador ha arrojado luz sobre las diferentes formas en que los miembros de un grupo, como un aula, contribuyen al acoso. Más allá de la díada intimidación-víctima, los incidentes de intimidación, generalmente, involucran a un grupo de espectadores: los asistentes se unen y ayudan al cabecilla a intimidar, mientras que los reforzadores permanecen alrededor y señalan su aprobación del acoso con risas o vítores (Vélez y Fernández, 2018, p. 175).

Este tercero en múltiples casos, es el sujeto más numeroso en el acto violento o conflicto, superando incluso el número de agresores o de víctimas; no 
obstante, como bien explica el autor en la cita anterior, será la conducta de este que aprueba o reprueba el acto, lo que pueda modificar lo que allí sucede, por ello no se puede compartir lo afirmado por Santoyo y Frías (2014) quien afirma que "finalmente, los observadores son aquellos que, sin estar vinculados de manera directa al acoso escolar, atestiguan y de alguna forma son partícipes de este" (p. 18). Mientras que el tercer observador sea un ser con condición humana, es claro que tendrá una preferencia por lo que allí sucede, sea por el sujeto o la situación según sus convicciones personales; por ello, si participa ese tercero en el acto violento -y dependiendo de su aprobación a uno u otro extremo de la situación en conflictos- tiene injerencia de forma activa o pasiva de dicha situación.

\section{Diseño y metodología}

Toda ejecución investigativa nace a partir de un cuestionamiento o pregunta de investigación y a su vez, la necesidad propicia la generación de objetivos a alcanzar por parte de los investigadores (Cifuentes et al., 2019). Por lo cual, el estudio base para la escritura de este artículo se ejecutó para dar respuesta a la pregunta ¿Cuáles son las diferencias que existen entre los vocablos conflicto y violencia, y cómo se definen los sujetos que participan en estos actos? Asimismo, se llevó a cabo bajo un paradigma cualitativo; puesto que se requería una comprensión de las concepciones y comportamientos de los participantes, en sus espacios académicos: "En primer lugar, este estudio tomó como referencia el paradigma cualitativo, por razón de qué era necesario la caracterización y comprensión detallada de la didáctica empleada por los participantes en sus diferentes facetas" (Quintero y Jaramillo, 2018, p, 88). Asimismo, se acoge bajo un enfoque hermenéutico por su característica interpretativa, para adquirir significado del escenario investigativo:

La primera característica a destacar en el enfoque hermenéutico es su motivación ontológica. Su interés primario está dirigido a desarrollar las preguntas elementales respecto al carácter propio y las condiciones generales de aquello qué es. Con el objetivo de plantear y esclarecer tales cuestiones, dicho enfoque se orienta, bajo influencia de la fenomenología, hacia el aparecer en la experiencia (Rubio, 2017, p. 283).

Por último, el tipo de investigación es un estudio de caso, ya que se percibió la situación investigada como algo diferencial que se representaba solo en ese contexto; así pues, Barquet y Machado (2017) establecen que "un estudio de caso es una metodología que se ajusta para analizar contextos particulares, que a su vez se enfrenta a un escenario que posee más variables que datos" (p. 11). Asimismo, la información que se recolecta es producto de la aplicación de algunos instrumentos, todo ello como consecuencia de la interpretación de matrices categoriales, partiendo de la triangulación de los 
datos producto de dichos instrumentos: la encuesta, la entrevista semiestructurada y el grupo focal.

Tabla 1. Muestra significativa - matriz categorial de análisis

\begin{tabular}{|c|c|c|c|c|c|}
\hline $\begin{array}{c}\text { Categorías } \\
\text { investigativas }\end{array}$ & $\begin{array}{c}\text { Relevancia } \\
\text { en el } \\
\text { instrumento } \\
1 . \text { (datos } \\
\text { relacionales } \\
\text { a la } \\
\text { categoría 1) }\end{array}$ & $\begin{array}{l}\text { Relevancia } \\
\text { en el } \\
\text { instrumento } \\
2 . \text { (datos } \\
\text { relacionales } \\
\text { a la } \\
\text { categoría 1) }\end{array}$ & $\begin{array}{c}\text { Relevancia } \\
\text { en el } \\
\text { instrumento } \\
\text { 3. (datos } \\
\text { relacionales } \\
\text { a la } \\
\text { categoría 1) }\end{array}$ & $\begin{array}{c}\text { Referencias } \\
\text { textuales } \\
\text { (investigaciones } \\
\text { previas en } \\
\text { relación a la } \\
\text { categoría) }\end{array}$ & $\begin{array}{c}\text { Visión crítica del } \\
\text { investigador a la } \\
\text { luz de los } \\
\text { resultados y las } \\
\text { investigaciones } \\
\text { análogas }\end{array}$ \\
\hline \multicolumn{6}{|l|}{ *Categoría \#1 } \\
\hline \multicolumn{6}{|l|}{ *Categoría \#2 } \\
\hline \multicolumn{6}{|l|}{ Violencia } \\
\hline \multicolumn{6}{|l|}{ *Categoría \#3 } \\
\hline \multicolumn{6}{|l|}{ Relación entre } \\
\hline \multicolumn{6}{|l|}{$\begin{array}{l}\text { conflicto y } \\
\text { violencia }\end{array}$} \\
\hline \multicolumn{6}{|l|}{ *Categoría \#4 } \\
\hline \multicolumn{6}{|l|}{ Rol del } \\
\hline \multirow{2}{*}{\multicolumn{6}{|c|}{ directivo ante el }} \\
\hline & & & & & \\
\hline \multicolumn{6}{|l|}{$\begin{array}{l}\text { conflicto y la } \\
\text { violencia }\end{array}$} \\
\hline \multirow{2}{*}{\multicolumn{6}{|c|}{$\begin{array}{l}\text { *Categoría \#5 } \\
\text { participación }\end{array}$}} \\
\hline & & & & & \\
\hline \multicolumn{6}{|l|}{ institucional en } \\
\hline la resolución de & & & & & \\
\hline onflict & & & & & \\
\hline
\end{tabular}

Fuente: Elaboración propia.

La muestra se tomó en el municipio de Copacabana-Antioquia (Colombia), en la Escuela San José de los Hermanos Terciarios Capuchinos, institución de rehabilitación de personas que han sufrido o generado violencia a otros. Para el criterio de selección (no probabilística), se buscó estudiantes que tuvieran un grado de instrucción entre segundo y tercer año de bachillerato; y, además, personal docente y administrativo que conociera el énfasis de la institución.

Tabla 2. Contextualización de las unidades de análisis

\begin{tabular}{llllll}
\hline $\begin{array}{l}\text { Institución } \\
\text { Educativa }\end{array}$ & Grados & Sección & Número de estudiantes & $\begin{array}{l}\text { Número de docentes } \\
\text { y administrativos }\end{array}$ \\
$\begin{array}{l}\text { Escuela San José } \\
\text { de los Hermanos }\end{array}$ & Séptimo y & "A” & Niñas & Niños & 5 \\
$\begin{array}{l}\text { Terciarios } \\
\text { Capuchinos }\end{array}$ & & 5 & 5 & \\
\hline \multicolumn{2}{l}{ Total de participantes } & & & & \\
\hline
\end{tabular}

Fuente: Elaboración propia. Selección no probabilística de participantes.

\section{Resultados y discusión}

En este apartado se evidencian los resultados cuantitativos arrojados por la encuesta semiestructurada aplicada durante los meses de octubre y noviembre del 2017 a un grupo de 10 estudiantes y 5 administrativos de la Institución Escuela de Trabajo San José (Copacabana - Antioquia).

A continuación, se dan a conocer una serie de resultados significativos sobre el estudio: Frente a la pregunta ¿Qué es la violencia escolar? Las 15 contestaciones rindieron una serie de respuestas que tienen en común la 
definición restringida de que se habló en acápites anteriores; es decir, no se definió la violencia, pero se explicó como sucede.

Sobre lo anterior, de los 15 participantes de las encuestas, el 19\% de los participantes la identificaron como el uso de la fuerza; un $33 \%$ como una forma de poder; y un $48 \%$ como un conflicto no resuelto; reforzando estas percepciones, así lo señaló también una de las unidades de análisis en las entrevistas:

La violencia es como ya pasar del conflicto al acto, como de una discusión a una agresión, a unos insultos o a una falta de respeto, a llevar como a hacer sentir mal a la otra persona o un choque entre los dos sujetos (Estudiante entrevistado 3, comunicación personal, 16 de octubre, 2017).

Se observa que, aunque tampoco podían definir con precisión el término violencia sí podían explicarla, en igual sentido otro participante entrevistado señaló que:

Violencia es la agresión frente a un interés particular, a una necesidad, a una condición, la violencia es transgredir la norma, imponer, generar dolor. Creo que se da cuando es un conflicto que se presenta mal, se presenta violencia (Administrativo entrevistado 2, comunicación personal, 16 de octubre, 2017).

A continuación, se muestra la gráfica que describe los datos expuestos sobre este resultado, al igual que refuerza la información que se dio a conocer a través de los testimonios de las unidades de análisis.

Figura 2. Definiciones de violencia: Encuesta aplicada a 15 participantes (10 estudiantes y 5 administrativos) de la Escuela San José de los Hermanos Terciarios Capuchinos, Medellín - Colombia, durante el mes de octubre en el año 2017.

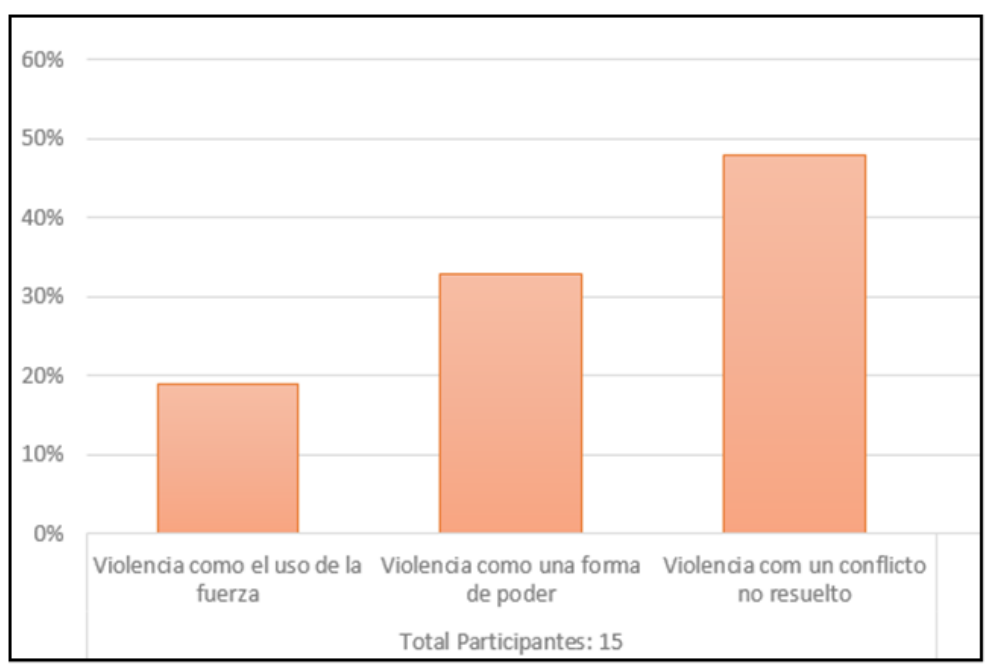

Fuente: Elaboración propia a parir de los resultados de la encuesta. 
Por otra parte, en el grupo focal los estudiantes explicaron que estas son algunas de las formas como se evidencia la violencia, señalaron que a veces con actos violentos se quiere obtener poder demostrando quién es el más fuerte, o buscar reconocimiento, como el protagonista en ese espacio escolar. Fueron claros en indicar -los 15 entrevistados- que 10 habían sido víctimas y agresores; es decir, unas veces unos abusaban de ellos, y como una forma de represalia ellos abusaban de otros, pero hoy reconocen estar equivocados y advierten que de tener claro que antes de llegar al acto violento, se presenta la diferencia o desacuerdo (conflicto); y del mismo modo, se podría intervenir y mediar en el asunto evitando resultados graves. Así lo deja claro el siguiente participante al explicar lo siguiente:

En mi experiencia, en mi básica en lo que se han presentado, son las dos, pero más que todo la violencia, pero en sí para llegar a la violencia se necesita pasar por un conflicto si me entiende, las dos, me ha pasado más la violencia (Estudiante entrevistado 7, comunicación personal, 16 de octubre, 2017).

Reconocieron que la violencia en la escuela afecta todo lo que sucede alrededor del proceso de formación. Y a pesar de todo ello, como ya se señaló, los sujetos que hacen parte de la muestra no pueden definir la palabra, sino explicarla a través de ejemplos que se han dado en su propia vida.

Si para los sujetos que han vivido en la violencia es difícil enmarcar esta palabra en un contenido propio, también lo es para los académicos; son situaciones como las antes señaladas las que sustentan la controversia que se presenta frente al tema, es claro que en el campo de las ciencias sociales, en cuanto a este vocablo se refiere, son múltiples los contenidos que se encuentran en la literatura, y al no tener este término una definición propia se han generado múltiples ejes de interpretación, siendo uno de los más actuales el que refiere a una definición restringida o amplia de la palabra violencia, tal y como señalan los ya citados Chuqilin y Zagaceta (2017); quienes hablaron de la interpretación amplia y restringida del fenómeno de violencia escolar, como consecuencia del vacío de la palabra, y ausencia de acuerdo académico frente al contenido del fonema, lo que ha creado que la palabra no se defina sino que se ejemplifique.

Es decir, hoy se caracteriza al sujeto de la violencia y se presentan las formas materiales de ella; a su vez, cada quien asume su propia definición (restringida o amplia). En este sentido, nadie desconoce que la violencia escolar -al igual que cualquier otra violencia- es el resultado de una conducta humana que en sus relaciones con otros se va a presentar; y seguramente, tendrá diferentes grados de dificultad, sin que existan fórmulas mágicas para resolverlo. Sin embargo, hay que partir de que las escuelas son espacios donde confluyen sujetos múltiples que deben encontrar un medio, una forma común para entenderse y poder desarrollar objetivos, algunos propios y otros comunes, pero todos deberán convivir por igual en ese espacio. 
Se encuentran, a su vez, hallazgos de otras investigaciones que coinciden con la que sirve de referente para este artículo, como sucedió con la realizada por Figueroa (2018), quien en un estudio de caso similar a este, encontró que se clasificó la violencia escolar a grandes rasgos en tres grupos: 1. Violencia directa que puede ser reconocida como física, verbal, psicológica, o por medios tecnológicos o coso.; 2. La violencia estructural que es la que desarrolla la institucionalidad frente al estudiante. 3. La violencia cultural, que son diferencias de tipo religioso, sexual o por conceptos culturales. Comprobando entonces esta teoría, se puede notar que coincide en su investigación con los resultados que se presentan en el estudio base para este texto, cuando precisan que una de las formas de violencia escolar es la agresión verbal, sin que ella misma sea la definición de la violencia, sino una forma de expresión de la misma; tal y como lo explica uno de los entrevistados en el grupo focal al señalar que:

La violencia ya es algo perjudicial, porque afecta, si no es física, mental, puede afectar a una persona, porque la violencia también se ve en la discriminación o se ve como, mucho en lo físico también en lo verbal, que una persona le dice o un apodo, también, o cuando le dice cosas que no le gusta, a la persona eso es ya como una violencia, que lo afecta (Administrativo entrevistado 5, comunicación personal, 13 de noviembre, 2017).

Los participantes de la muestra tienen claro que la violencia escolar los perjudica; saben la afectación que puede generar en el otro, pero lo que no encuentran es la forma de eliminar el ciclo de "no repetirlo", advirtiendo que la violencia tiene múltiples causas y esas múltiples causas están en lo que "no entiendo", "no comparto" o "no acepto del otro". En ese sentido, existieron aportes muy importantes en esta investigación, uno de los participantes explicó en el grupo focal con gran claridad que, a pesar de todo, con estos hechos nadie gana: "nadie va a ganar en el conflicto, todos van a salir perdiendo, su libertad o cualquier tipo de cosa" (Administrativo entrevistado 4, comunicación personal, 13 de noviembre, 2017) siempre existen antecedentes previos al hecho violento mismos, que en este ejercicio se encontraron y se clasificaron como conflicto.

Partiendo de lo expuesto por los entrevistados, el tema del conflicto adquiere mayor relevancia que la misma violencia, si se entiende que la violencia tiene un precedente en el conflicto, por ello se debe entender qué es el conflicto, recordando que los académicos lo tienen entendido como las diferencias o los desacuerdo; y en forma casi unánime, 13 de los 15 entrevistados lo identifican de igual forma, como un desacuerdo con otro, como un mal entendido con otro, como una "no comunicación" con otro.

A estas alturas de la investigación, es claro que ni el conflicto ni la violencia se presentan en soledad, siempre se presentan en relaciones con otro, frente a otro, por circunstancias particulares con otro. Por lo tanto, toda diferencia se 
da con otro individuo, así lo expresó claramente uno de los entrevistados al afirmar que; "el conflicto es como un problema que uno tiene con otra persona, algo que le incomode a uno" (Estudiante entrevistado 5, comunicación personal, 16 de octubre, 2017), por consiguiente, si se resuelve, desaparece el conflicto y necesariamente, no habrá acto violento. Este descubrimiento coincide con la postura de Lapponi (2013), quien precisa que: "conflicto es toda actividad en que unos hombres contienden con otros por la consecución de un los objetivo/s. Implica desarmonía, incompatibilidad, pugna entre dos partes" (p. 92). Los grados de incompatibilidad no son más que las mismas diferencias o desavenencias, la cuales pueden presentarse por múltiples factores; así pues, durante la aplicación de técnicas de recolección, se encontró que estas diferencias se pueden dar por razones múltiples, que van desde sexualidad, pertenecer a ciertos barrios (fronteras invisibles), entre otras:

Pues más que todo yo he sufrido porque por mi barrio se ven mucho las fronteras invisibles y el conflicto de allá quería que yo me uniera a ellos, yo la única alternativa que tuve fue venirme para acá para Machado porque la verdad nunca me ha gustado eso, nunca he sido así (Estudiante entrevistado 10, comunicación personal, 16 de octubre, 2017).

Y seguramente también habrá otro tipo de violencias, tal vez no tan visibles pero que en la escuela siguen generando debate o desacuerdo, como será el de pertenecer o no a algún credo o religión, diferencias de conocimiento siempre con certeza; por ende, aquel que sabe más o menos que otro, suele ser segregado del grupo, y no ha de faltar las fútiles discriminaciones que se establecen por raza y color de piel, extremadamente soterradas pero latentes en la contemporaneidad. Muchos de los entrevistados manifestaron que la violencia es una de las formas de demostrar poder en un entorno específico; y que, por ello, muchas veces no importa el hecho de obtener el reconocimiento ante el grupo social al cual se pertenezca, incluida la escuela. Por eso -y en aras de intervenir esta situación- los aportes de Pérez (2001) son muy importantes cuando explica que:

A medida que los currículos incluyan contenidos racionales, afectivos, morales y sociales, y que en los centros se trabaje de forma sistemática e intencional, estas vertientes, se irá produciendo, aunque sea ligeramente, una mudanza individual y colectiva a favor de la convivencia (p. 316).

Es decir, en los currículos actuales hay que incluir conceptos prácticos y contextualizados que mejoren la convivencia, y dentro de los cuales habría que regresar a concepciones que reivindiquen valores como el respeto por las diferencias. Por lo cual, la escuela debe ser mirada en contextos reales y dentro de estos, aceptar la existencia del conflicto e incluirlo en el desarrollo de sus currículos, como una forma de prevención de la violencia. En este 
sentido, uno de los administrativos señala, "Somos responsables, toda la comunidad educativa debe tomar una postura de responsabilidad frente a la mediación de situaciones y de conflictos" (Administrativo entrevistado 1, comunicación personal, 13 de noviembre, 2017). Por ello, una forma de modificar la violencia escolar es construyendo currículos que incluyan la realidad del conflicto; así pues, y conforme lo explican el ya citado Ayala (2015) "Resulta muy importante que los centros educativos, la familia y la sociedad en general, dediquen sus mayores esfuerzos en el desarrollo de una gestión constructiva del conflicto" (p. 17).

Ahora, frente a la clasificación de los sujetos, los estudiantes durante la sesión del grupo focal fueron quienes primero identificaron que esta clasificación había mutado de una teoría dual a una tríada. Este es un ejemplo puntual de cómo lo describieron: "Serían víctima y victimario, y las personas que hay afuera como observando yo las llamaría espectadores, porque ellos hacen parte de la situación" (Estudiante entrevistado 3, comunicación personal, 13 de noviembre, 2017), de los 15 sujetos entrevistados, con diferencias leves, la mayoría afirmó que los sujetos del acto violento son tres, no dos como anteriormente se solía pensar. En el grupo focal se explicó con claridad, que no solo son las víctimas y los agresores los sujeto que participan del acto violento; para ellos el mayor número de sujetos son los terceros, los observadores, los aparentemente no intervinientes en el acto violento. Sobre esta situación, la mayoría de los participantes explicaron que existen otros, a quienes llamaron "observadores" o "espectadores", y explicaron que los otros siempre tomaban partido en el acto violento a favor de unos u otros, tanto de forma activa como pasiva, pero siempre estaban ahí aportando al acto, por eso ellos también hacen parte del acto.

Las respuestas dadas por los entrevistados demuestran que hoy se ha modificado el número de participantes en el acto violento, que con la evolución del fenómeno ha mutado de dos partes a tres sujetos y por ello, ya la relación no es dual sino que generó una triada de sujetos, donde cada uno de ellos tiene actuares y conductas definidas, y donde ese tercero puede hacer parte o no de los sujetos de la escuela; de manera que, ese tercero puede ser un representante cualquiera de las otras esferas sociales, y por consiguiente, hoy la intervención de este fenómeno requiere la concurrencia de todos los actores sociales. Investigaciones recientes han descubierto que estos sujetos ocupan estas posiciones de forma alternada, como Rodney y Garcia (2015), quienes señalan que:

En investigaciones realizadas en una escuela secundaria básica a una población de 146 miembros del estudiantado, 66 muchachas y 79 muchachos, en edades comprendidas entre 11 y 14 años, se constató que los miembros del estudiantado ocupan diferentes roles en situaciones de violencia (víctimas, agresores y espectadores) (p.170). 
Sin embargo, nada de esto sucede en forma aislada; lo que acontece en la escuela está unido a lo que sucede en la familia y en la sociedad. No se puede presumir que un asunto de tal magnitud se pueda observar alternadamente, los contextos sociales intervienen en diferentes etapas de crecimiento personal; por lo tanto, el debate de que "la violencia es aprendida" no ha perdido aún vigencia, y no se puede intervenir un problema como estos de manera individual, o buscar respuesta en contextos únicos y excluyentes en un sentido similar, a lo que anota Ayala (2015) en el sentido de precisar que:

El aula escolar se considera un espacio de construcción de identidades, sin embargo, lo que sucede en éstas es un reflejo de lo que sucede afuera, ya sea en las relaciones familiares, en las calles, en la comunidad, en el país. Por lo tanto, las interrelaciones que se dan entre el alumnado, se producen y reproducen a partir de las experiencias previas de cada uno(as) en relación con el mundo externo, pero también con lo subjetivo del grupo (p. 495).

Para concluir, se hace imperativa la comprensión de lo que es violencia y conflicto; asimismo, el entendimiento sobre los sujetos que participan en estos actos, para de esta forma hacer una intervención certera en los momentos que se requiera.

\section{Conclusiones}

Terminada la investigación emergen claras resoluciones. Una de ellas es que no existe una forma concreta y clara de definir la palabra violencia; existen múltiples enfoques, siendo uno de los más interesantes el que refiere a la definición amplia o restringida del vocablo en cuestión.

De igual forma, existe una manera clara de definir el conflicto como una diferencia entre sujetos, sobre lo que no hay claridad es en el hecho de que la violencia está precedida del conflicto; sin embargo, esto queda como prospectiva y debería ser objeto de otra investigación análoga.

En lo tocante a los sujetos, ya no solo se pueden hacer referencia a víctimas y agresores, pues hoy se debe hablar de víctima, agresor y observador. Esta última figura se caracteriza por el número de sujetos que hacen de espectadores durante los actos de violencias; del mismo modo, el número de observadores casi siempre suele ser un sujeto plural-mayor.

Finalmente, en este análisis fue evidente que sí se interviene el conflicto, se evita la violencia; entendiendo que lo primero está contenido en lo segundo, y que, por ser el número de observadores, mayores al número de sujetos que participan de forma directa en el acto, entonces será a estos terceros a quien deba dedicarse ahora un mayor análisis para la solución, partiendo como siempre, desde la conformación de mallas curriculares en contextos reales. No obstante, estos dos puntos serán objeto de nuevos estudios académicos. 


\section{Referencias Bibliográficas}

Álvarez, G. D., Dobarro, A., Rodríguez, C., Núñez, J. C. y Álvarez, L. (2013). El consenso de normas de aula y su relación con bajos niveles de violencia escolar. $\begin{array}{lllll}\text { Infancia } y & \text { Aprendizaje, 36(2), 199-217. DOI: }\end{array}$ https://doi.org/10.1174/021037013806196229

Arellano, N. (2007). La violencia escolar y la provencion del conflicto. Orbis. Revista Cientifica Ciencias Humanas, 3(7), 23-45. Recuperado de: https://bit.ly/2ZRVhqi

Artavia, G. J. M. (2012). Manifestaciones de violencia explícita o evidente durante el desarrollo del recreo escolar. Actualidades Investigativas en Educación, 12(2). 1-29. Recuperado de: https://revistas.ucr.ac.cr/index.php/aie/article/view/23173

Ayala, C. M. D. (2015). Violencia escolar. Un problema complejo. RAXIMHAI, 11(4), 493-509. DOI: https://doi.org/10.35197/rx.11.01.e2.2015.36.ma

Aznar, I., Cacares, M. P. y Hinojo, F. J. (2018). Estudio de la violencia y conflictividad escolar en las aulas de educacion primaria a traves de un cuestionario de clima de clase. El caso de las provincias de Córdoba y Granada (España). Electrónica Iberoamericana sobre Calidad, Eficacia y Cambio en la Educacion, 5(1), 164-177. Recuperado de: https://bit.ly/2ZQeNDr

Barquet, E. A. G. y Machado, O. J. A. (2017). El estudio de casos: una metodología efectiva para la investigación empresarial. Revista Espacios, 38(51), 10-16. Recuperado de: https://bit.ly/2BqccHa

Bonacera, E. O. (2015). Lecciones para el posconflicto: ¿Por qué el fin del conflicto armado en María la Baja, Bolívar (2006-2014) no puso fin de la violencia?. Revista Palobra," palabra que obra", 15(15), 260-271. DOI: https://doi.org/10.32997/2346-2884-vol.15-num.15-2015-847

Cavas, C. M. J. (2009). La utilidad de la mediacion como estrategia de resolucion y prevencion de conflictos en el ámbito escolar. Información Psicològica, (95), 1526. Recuperado de: https://bit.ly/2ZQ9uE2

Cerezo, R. F. (2015). Agresores y víctimas de bullying. Desigualdades de genero en la violencia entre escolares. Información Psicológica, (94), 48-59. Recuperado de: https://bit.ly/3hr1lfh

Chaux, E. (2011). Multiples perspectivas sobre un problema complejo:comentarios sobre cinco investigaciones en violencia escolar. PSYKHE, 20(2), 79-86. DOI: https://doi.org/10.4067/S0718-22282011000200007

Chávez, A. S. X. (2018). La mediación escolar y las relaciones interpersonales del estudiantado de primer curso de bachillerato general Unificado, paralelos $A$ y $B$, de la Unidad Educativa Indoamérica, cantón Ambato (Tesis de Pregrado). Universidad Técnica de Ambato, Ambato, Ecuador. 
Chuquilin, C. J. y Zagaceta, S. M. (2017). La violencia en las escuelas desde la perspectiva de sus actores. El caso de una escuela de segundaria de la ciudad de Mexico. Revista Educación, 41(2), 1-19. DOI: https://doi.org/10.15517/revedu.v41i2.21751

Cifuentes, L. M., Martínez, G. L., Jaramillo, V. B., Cardona, M. N. y Jiménez, H. S. (2019). Entre la oferta y la demanda: el idioma inglés visto como una barrera invisible que interfiere en el acceso a la labor docente de las profesionales en educación inicial. In Augusto Silva (Ed.) Retos y Desafios: Perspectivas de la Educación y la Empresa (págs. 8-21). Bucaramanga: Editorial EIDEC.

Figueroa, H. M. R. (2018). La violencia escolar en el bachillerato. Un estudio de caso. Caleidosocopio-Revista Semestral de Ciencias Sociales y Humanidades, 18(33), 15-43. DOI: https://doi.org/10.33064/33crscsh216

Flores, G. L. M. y Retamal, S. J. A. (2011). Clima escolar y gestion compleja del conocimiento; desafio para la investigacion educativa y la politica pública de la violencia escolar. Magis, 4(8), 319-338. Recuperado de: https://bit.ly/32K4yT3

Gomez, N. A. (2013). Bullying: El poder de la violencia. Una prespectiva cualitativa sobre acosadores, víctimas en la escuela primaria de Colima. Revista Mexicana de Investicaciones Educativa, 18(58), 839-870. Recuperado de: https://bit.ly/3ePThDd

Jano, B., De Caso, A. M. y Navas, G. (2012). Violencia escolar. Ciberbulling en redes sociales. International Journal of Developmental and Educational Psychology, 1(1), 717-724. Recuperado de: https://bit.ly/32QQpDX

Lapponi, S. F. (2013). Resolución de conflictos en la escuela: una herramienta para la cultura de paz y la convivencia. Contextos Educativos. Revista de Educación, (3), 91-106. DOI: https://doi.org/10.18172/con.466

Olweus, D. (1989). Prevalence and incidence in the study of antisocial behavior: definitions and measurements. Dordrecht: Springer. DOI: https://doi.org/10.1007/978-94-009-1001-0_9

Pérez, F. M. D., Álvarez, B. J. A., Molero, M. D., Gazquez, J. J. y López, V. M. A. (2014). Violencia escolar y rendimiento académico (VERA): aplicación de realidad aumentada. European Journal of investigation in health, psychology and education, 1(2), 71-84. DOI: https://doi.org/10.30552/ejihpe.v1i2.19

Rodríguez, G. A. M., Solá, M. T. y López, M. J. A. (2017). Centro Juan XXIII de Granada como modelo referente en la resolución de conflictos y mejora del clima escolar. Interacções, 13(43), 97-115. DOI: https://doi.org/10.25755/int.12032

Pérez, V. M. O. (2001). Convivencia escolar: problemas y soluciones. Revista Complutense de Educación, 12(1), 295-318. DOI: http://dx.doi.org/10.5027/psicoperspectivas-vol18-issue1-fulltext-1486 
Prieto, M., Carrillo, J. y Jiménez, J. (2005). La violencia escolar: Un estudio en el nivel medio superior. Revista Mexicana de Investigación Educativa, 10(27), 1027-1045. Recuperado de: https://bit.ly/2ZSkYXC

Quintero, A. S. y Jaramillo, V. B. (2018). La didáctica en profesores de educación parvularia o preescolar: Los casos de Colombia y Chile. En Serna Édgar (Ed.) Revolución en la Formación y la Capacitación para el Siglo XXI (pp. 86-93). Medellín: Editorial Instituto Antioqueño de Investigación.

Rodney, R. Y. y García, L. M. (2015). Políticas públicas sobre violencia escolar en Cuba: entre lo jurídico y la realidad. Revista Sexología y Sociedad, 21(1), 146180. Recuperado de: https://bit.ly/2ZOPdPq

Rubio, R. (2017). The regent philosophy of the image a critical analysis of the current debate and a consideration of possible contributions. Ideas y Valores, 66(163), 273-298. DOI: https://doi.org/10.15446/ideasyvalores.v66n163.51068

Santoyo, C. D. y Frías, S. M. (2014). Acoso escolar en México: actores involucrados y sus características. Revista Latinoamericana de Estudios Educativos Mexico, 44(4), 13-41. Recuperado de: https://bit.ly/2ONyqpA

Sinning, E. R. (2006). Las nuevas liturgias patrióticas: Celebraciones en Santa Marta, con ocasión de la elección y posesión de tres presidentes neogranadinos. Palobra: Palabra que obra, (7), 76-91. DOI: https://doi.org/10.32997/2346-2884vol.7-num.7-2006-167

Torres, E., Palermo, Y. C. y Bedoya, C. P. (2018). La infancia y los niños en tiempos de guerra: el caso de Nicaragua, El Salvador y Guatemala. Palobra: Palabra que obra, (18), 194-215. DOI: https://doi.org/10.32997/2346-2884-vol.0-num.182018-2171

Vélez, S. C. y Fernández, J. A. Á. (2018). Influencia de los observadores sobre la agresión y el sentimiento de maltrato escolar. Psychology, Society, \& Education, 10(2), 173-187. DOI: https://doi.org/10.25115/psye.v10i2.1021 